This is the author's final, peer-reviewed manuscript as accepted for publication. The publisher-formatted version may be available through the publisher's web site or your institution's library.

\title{
Q-space analysis of scattering by small irregular particles
}

C. M. Sorensen, E. Zubko, W. R. Heinson, A. Chakrabarti

\section{How to cite this manuscript}

If you make reference to this version of the manuscript, use the following information:

Sorensen, C. M., Zubko, E., Heinson, W. R., \& Chakrabarti, A. (2014). Q-space analysis of scattering by small irregular particles. Retrieved from http://krex.ksu.edu

\section{Published Version Information}

Citation: Sorensen, C. M., Zubko, E., Heinson, W. R., \& Chakrabarti, A. (2014). Qspace analysis of scattering by small irregular particles. Journal of Quantitative Spectroscopy \& Radiative Transfer, 133, 99-105.

Copyright: @ 2013 Elsevier Ltd

Digital Object Identifier (DOI): doi:10.1016/j.jqsrt.2013.07.020

Publisher's Link: http://www.sciencedirect.com/science/article/pii/S0022407313003130

This item was retrieved from the K-State Research Exchange (K-REx), the institutional repository of Kansas State University. K-REx is available at http://krex.ksu.edu 
Q-Space Analysis of Scattering by Small Irregular Particles

C. M. Sorensen ${ }^{\mathrm{a}, *}$, E. Zubko ${ }^{\mathrm{b}}$, W. R. Heinson ${ }^{\mathrm{a}}$ and A. Chakrabarti ${ }^{\mathrm{a}}$

${ }^{\mathrm{a}}$ Department of Physics, Kansas State University, Manhattan KS 66506 USA

b Department of Physics, P.O. Box 64, FI-00014 University of Helsinki, Finland and Astronomical Institute of V.N. Karazin Kharkov National University, 35 Sumskaya St., Kharkov, 61022, Ukraine

Keywords:

Light scattering

Q-space analysis

Phase function

Irregularly shape particles

* Corresponding author at: Department of Physics, Kansas State University, Manhattan KS 66506 USA.E-mail address: sor@phys.ksu.edu

Abstract. This paper applies the Q-space analysis method to the scattering phase function of small irregular particles. Q-space analysis involves plotting the scattered intensity versus the magnitude of the scattering wave vector $\mathrm{q}=(4 \pi / \lambda) \sin (\theta / 2)$ on a double $\log$ plot. Four types of irregularly shaped particles were studied: strongly damaged spheres, rough surface spheres, pocked spheres, and agglomerated debris particles. The angular scattering phase function was calculated using the discrete dipole approximation (DDA). The Q-space analysis uncovered power law descriptions of the scattering as it has previously for aggregates, spheres and dusts, although in some situations the description is marginal. It also showed that the forward scattering lobe has Rayleigh functionalities on size and refractive index. These results imply that Q-space analysis can yield a comprehensive description of scattering in terms of power laws with quantifiable exponents for a wide variety of particle shapes. However, a theoretical explanation of the power laws and the values of the numerical exponents is lacking. 


\section{Introduction.}

The description of light scattering by particles evolves from the simple Rayleigh limit which applies to particles much smaller than the wavelength of light, to spherical particles of arbitrary size and refractive index describable by the classical Mie equations, to a vast variety of complex, irregular geometries such as aggregates, spheroids, and innumerable random shapes that appear in soot, mineral dusts, volcanic ash, snow and ice crystals, and biological microorganisms. The foremost agenda in the light scattering literature for the past few decades has been how to describe and calculate scattering and absorption by irregularly shaped particles. Significant computational advances have been made, and very useful experimental programs have allowed for comparison with computation. However, a simple description encompassing all shapes has yet to be obtained. In this paper we continue our investigation of the facility of the Qspace analysis [1-4] to provide a comprehensive and quantitative description of the scattering phase function for all shapes. Q-space analysis is applied to discrete dipole approximation (DDA) scattering calculation results of Zubko and coworkers [5- 7] for four different particle morphologies: strongly damaged spheres, rough surface spheres, pocked spheres, and agglomerated debris particles. A coherent description of scattering involving power laws emerges which indicates a potential unification of these irregular particles with spheres, spheroids, dusts and aggregates.

\section{Q-space Analysis.}

Q-space analysis involves plotting the scattered intensity as a function of the magnitude of the scattering wave vector

$$
\mathrm{q}=2 \mathrm{k} \sin (\theta / 2)
$$

on double-log plots [1]. Here $\mathrm{k}=2 \pi / \lambda$ where $\lambda$ is the wavelength and $\theta$ is the scattering angle. The units of $\mathrm{q}$ are inverse length. Plotting in this manner yields a significant difference from plots of the same intensity data against linear $\theta$.

With Q-space analysis, aggregates show power laws in the scattered intensity at large $\mathrm{q}$ with negative slopes equal to the aggregate fractal dimension [8]. As q decreases, the power law transforms through a curving Guinier [9] regime near $\mathrm{qR}_{\mathrm{g}} \approx 1$, where $\mathrm{R}_{\mathrm{g}}$ is the cluster radius of gyration, which is dominated by simple diffraction. As q approaches zero, the Guinier regime gives way to a q-independent Rayleigh regime.

When the Q-space analysis is applied to the scattered intensity from spheres of arbitrary size and refractive index, the Mie solution [10], power laws again appear in the envelops of the plots (ignoring the "ripples" and the enhanced backscattering) and a simple description results [1-4]. One finds a quasi-universality of plots for spheres of wide ranging size and refractive index if the so-called phase shift parameters are equal. The phase shift parameter is [11]

$$
\rho=2 \mathrm{kR}|\mathrm{m}-1|
$$

where $\mathrm{m}$ is the refractive index and $\mathrm{R}$ is the sphere radius. As for aggregates, at small $\mathrm{q}$ the scattered intensity in the forward lobe Rayleigh regime is constant as $\mathrm{q}$ approaches 0 and a Guinier regime occurs near $\mathrm{qR} \approx 1$. For $\mathrm{qR}>1$ the scattered intensity falls off with a rough power law as $(\mathrm{qR})^{-2}$ until this functionality crosses over at $\mathrm{qR} \approx \rho$. For $\mathrm{qR}>\rho$ the scattered intensity falls off as $(\mathrm{qR})^{-4}$. If $\rho<1$, there is no -2 power law and the Guinier regime crosses over directly to the -4 power law with increasing q. Recently the Q-space analysis was applied [12] to 
experimental data for dusts $[13,14]$. These dusts are solid particles that lack spherical symmetry. Some have smaller dusts on their surface. Once again Q-space analysis yielded power laws and all four types of dusts studied showed the same power law exponent. In summary, so far the tally has aggregates, arbitrary spheres as described by Mie scattering and non-spherical irregular dusts all show power laws with quantifiable exponents under Q-space analysis. These results compel us to apply the Q-space analysis to yet other shapes and refractive indices to build an extensive empirical base that could both yield a comprehensive description of light scattering by all shapes and lead to an explanation of the power laws. Below we show that Q-space analysis also yields quantifiable power laws when applied to calculated scattering results for irregularly shaped particles.

\section{Application of Q-space Analysis to Irregular Spheres.}

Zubko and coworkers [5-7] used DDA [e.g., 15] to calculate the scattering for four different types of irregular spheres: strongly damaged spheres, rough surface spheres, pocked spheres, and agglomerated debris particles. For each type three refractive indices were used: $\mathrm{m}=$ $1.313,1.5+0.1 \mathrm{i}$ and $1.6+0.0005 \mathrm{i}$. The size parameter $\mathrm{x}=\mathrm{kR}$, where $\mathrm{R}$ is the radius of the circumscribing sphere of the particle, ranged from 2 to 14 with steps of 2 . For a wavelength of $0.633 \mu \mathrm{m}$, these values correspond to radii of $\mathrm{R}=0.2$ to $1.4 \mu \mathrm{m}$. It is important to stress that the light scattering response of irregularly shaped particles for each set of $\mathrm{x}$ and $\mathrm{m}$ was averaged over sample particles and their orientations. We considered a minimum of 500 sample particles. Each sample particle was computed for only one random orientation of the incident electromagnetic wave, but was azimuthally averaged over 100 scattering planes, evenly distributed around the propagation direction of the incident light. We continued the averaging over additional sample particles until fluctuations of the standard deviation of the degree of linear polarization over the entire range of the scattering angle $\theta$ was less than $1 \%$; whereas, the actual number of sample particles considered for each set of parameters often exceeded 500 . Thus, the data used in the present investigation were quite statistically reliable. More detail on the DDA calculation, the particle shapes and the averaging over scattering planes and control of the averaging quality can be found in work of Zubko et al. [5- 7].

\subsection{Strongly damaged spheres.}

To start the analysis the real space structure of some of the particles was created by replacing the dipoles that make up the particle for the DDA calculation with points on a lattice. Then the structure factor of the particle was calculated as the square of the Fourier transform of this real space structure [8]. Figure 1a shows the structure factor for three of the strongly damaged spheres plotted versus $\mathrm{qR}_{\mathrm{g}}$, where $\mathrm{R}_{\mathrm{g}}$ is the radius of gyration of the particle. This represents the scattering in the $\mathrm{m} \rightarrow 1.00, \rho \rightarrow 0$ limit, i.e. the Rayleigh-Debye-Gans limit. A prominent power law is seen with an exponent of -4 (the upswing at large $\mathrm{qR}_{\mathrm{g}}$ is an artifact due to the finite separation of the dipoles). This is the Porod value for a sphere $[8,9,16]$ and in that respect is consistent with the sphere morphology, albeit perturbed. It is well known that scattering from a homogeneous sphere in the $\mathrm{m} \rightarrow 1.00(\rho \rightarrow 0)$ limit is described by the Rayleigh-Debye-Gans (RDG) [11] formula with a $\mathrm{q}^{-4}$ envelop at large q. Q-space analysis of Mie scattering by spheres $[1,2]$, reviewed above, showed that spherical particle scattering evolved from this RDG limit with a power law exponent of -4 to exponent -2 and -4 regimes with increasing $\mathrm{m}$. The Q-space plots for strongly damaged spheres are shown in Fig. 1, parts b, $\mathrm{c}$ and $\mathrm{d}$. Rather than the variable $\mathrm{q}$ the dimensionless variable $\mathrm{qR}=2 \mathrm{x} \sin (\theta / 2)$ is used (note that the maximum value of $\mathrm{qR}$ is $2 \mathrm{kR}=2 \mathrm{x}$ ). Moreover, the scattered intensities are shifted by multiplicative factors to avoid overlapping of the plots. For each refractive index the following 
features are seen:

1. A q-independent Rayleigh regime appears when $\mathrm{qR}<1$.

2. A Guinier regime near $\mathrm{qR} \approx 1$.

3. A prominent "hump" in the range $1 \leq \mathrm{qR} \leq 3.5$.

4. A minimum near $\mathrm{qR} \approx 3.5$.

5. A power law regime when $\mathrm{qR}>3.5$ with exponents that tend to decrease with increasing refractive index and size parameter. The power law is the envelop of a series of interference ripples and for the two smallest refractive indices appears to include the hump down to $\mathrm{qR} \approx 1$.

6. Enhanced back scattering at the large $\mathrm{qR}$ end where $\mathrm{qR}$ is equal to twice the size parameter, $2 \mathrm{x}$.

These features including the power laws are similar to the numerous others observed for aggregates, spheres and dusts via Q-space analysis as described above.

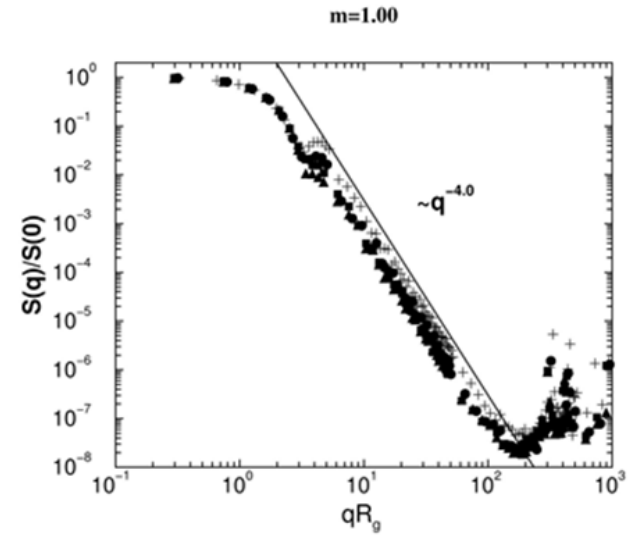

a.

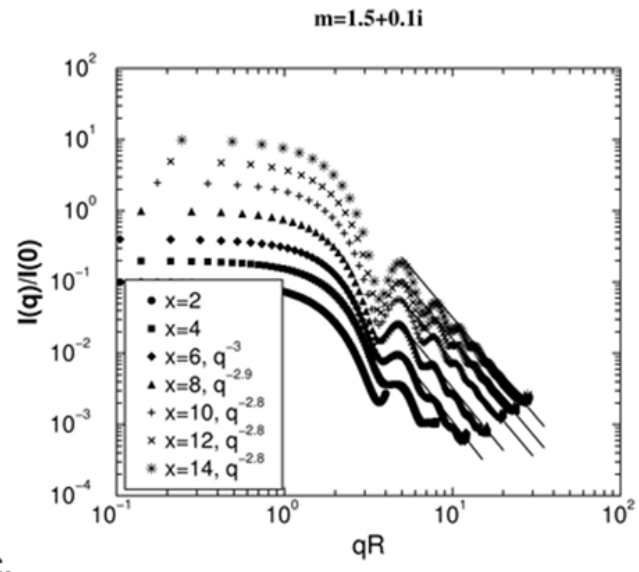

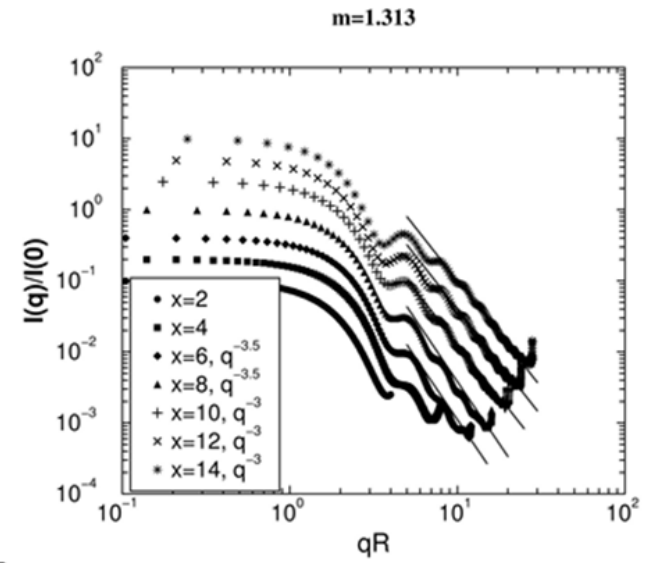

b.

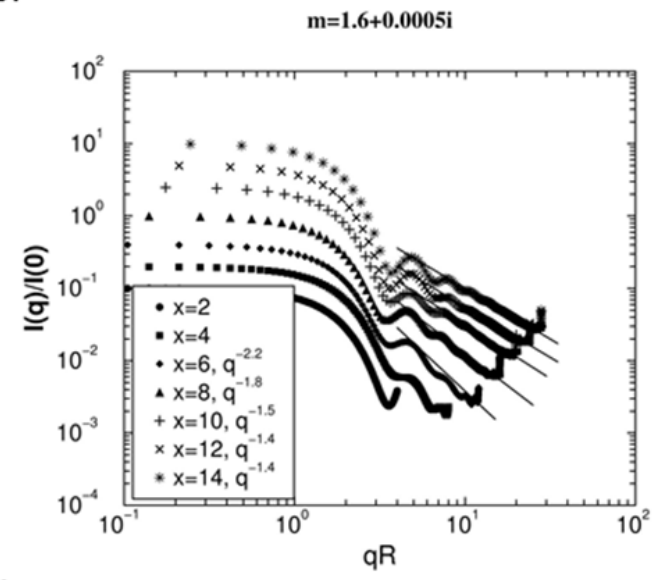

Figure 1. Q-space analysis of the scattering by strongly damaged spheres. Part a shows the $\mathrm{m} \rightarrow 1.00$, Rayleigh-Debye-Gans limit, the structure factor. Parts b, c and d are with refractive indices, $\mathrm{m}$, as marked for a range of size parameters, $\mathrm{x}$. Thin straight lines represent power laws with $\mathrm{q}$ as described in the legend. Plots are intensity shifted to avoid overlap. 
We conclude that while the -4 regime remains at $\mathrm{m}=1.00$, i.e. the structure factor, for strongly damaged spheres, the $\mathrm{m}>1$ exponents of Fig. $1 \mathrm{~b}, \mathrm{c}$ and $\mathrm{d}$ are significantly different than for homogeneous spheres and only one power law is seen, not two. Note also the small dip near $\mathrm{qR}_{\mathrm{g}} \approx 3$ in the structure factor similar to that seen in the scattering results. This dip, and similar ones to be seen below, might be due to a structural artifact in the particle creation method.

3.2 Rough surface spheres. The Q-space plots for rough surface spheres are shown in Fig.2. Similar to the strongly damaged spheres case above, Fig. 2 shows all six characteristics delineated above including Rayleigh, Guinier and power law regimes. However, for these particles the power law description is not very good, and the ripple structure dominates, but it is still more descriptive than plotting versus linear scattering angle which yields non-descript curves. The structure factor in Fig. 2a again shows the Porod $\mathrm{q}^{-4}$ behavior and the idiosyncratic $\operatorname{dip}$ at $\mathrm{qR}_{\mathrm{g}} \approx 3$.

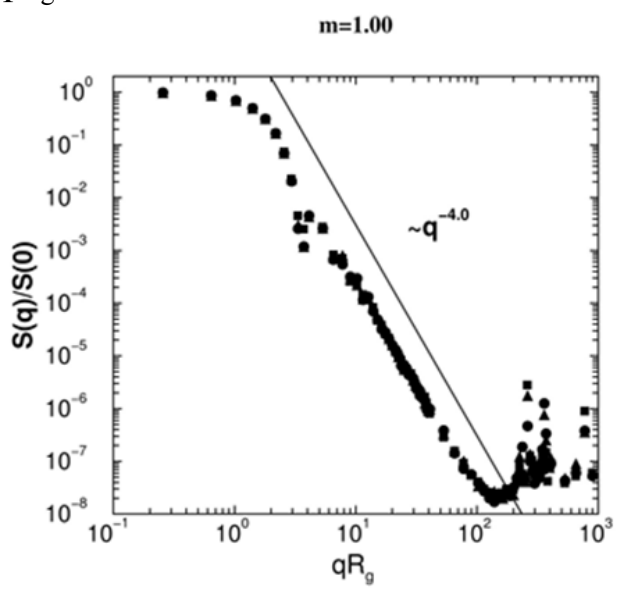

a.

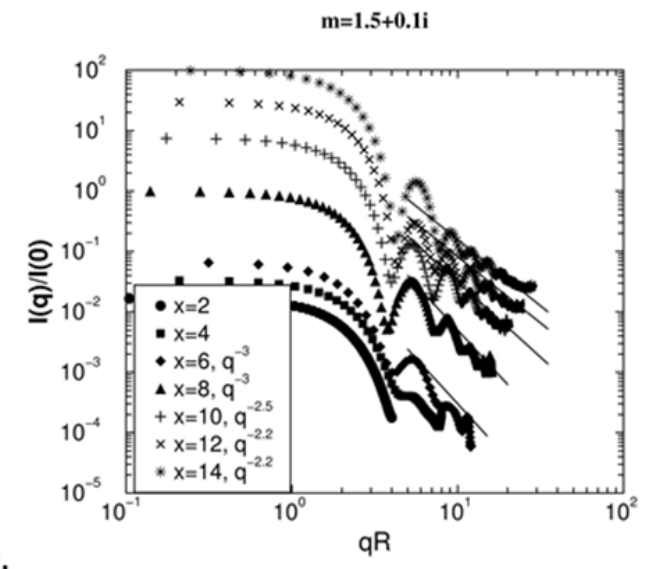

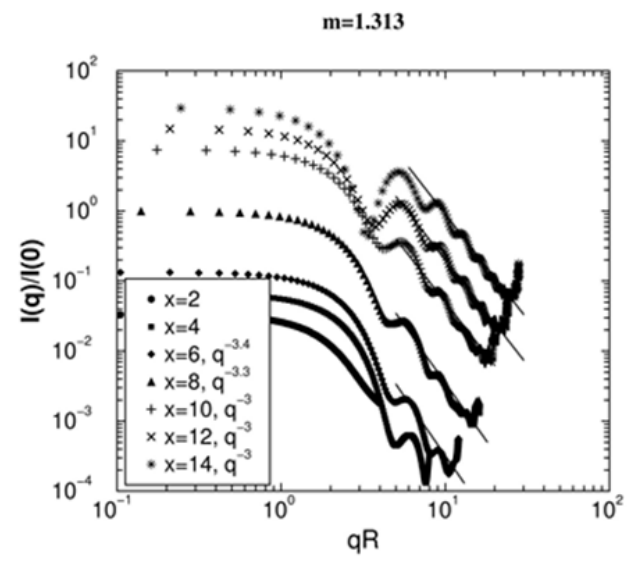

b.

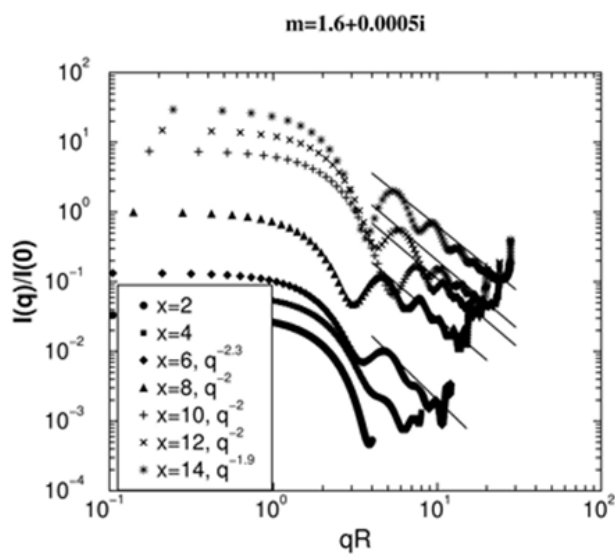

Figure 2. Q-space analysis of the scattering by rough surface spheres. Part a shows the $\mathrm{m}$ $\rightarrow 1.00$, Rayleigh-Debye-Gans limit, the structure factor. Parts b, c and d are with refractive indices, $\mathrm{m}$, as marked for a range of size parameters, $\mathrm{x}$. Thin straight lines represent power laws with $\mathrm{q}$ as described in the legend. Plots are intensity shifted to avoid overlap. 
3.3 Pocked spheres. The Q-space plots for pocked spheres are shown in Fig. 3. Similar to the strongly damaged spheres case above, Fig. 3 shows all six features including Rayleigh, Guinier and definite power law regimes. The ripple structure is weak. Figure $3 \mathrm{~d}$ shows the square of the Fourier transform of the real space structure for three of the pocked spheres. Once again in the
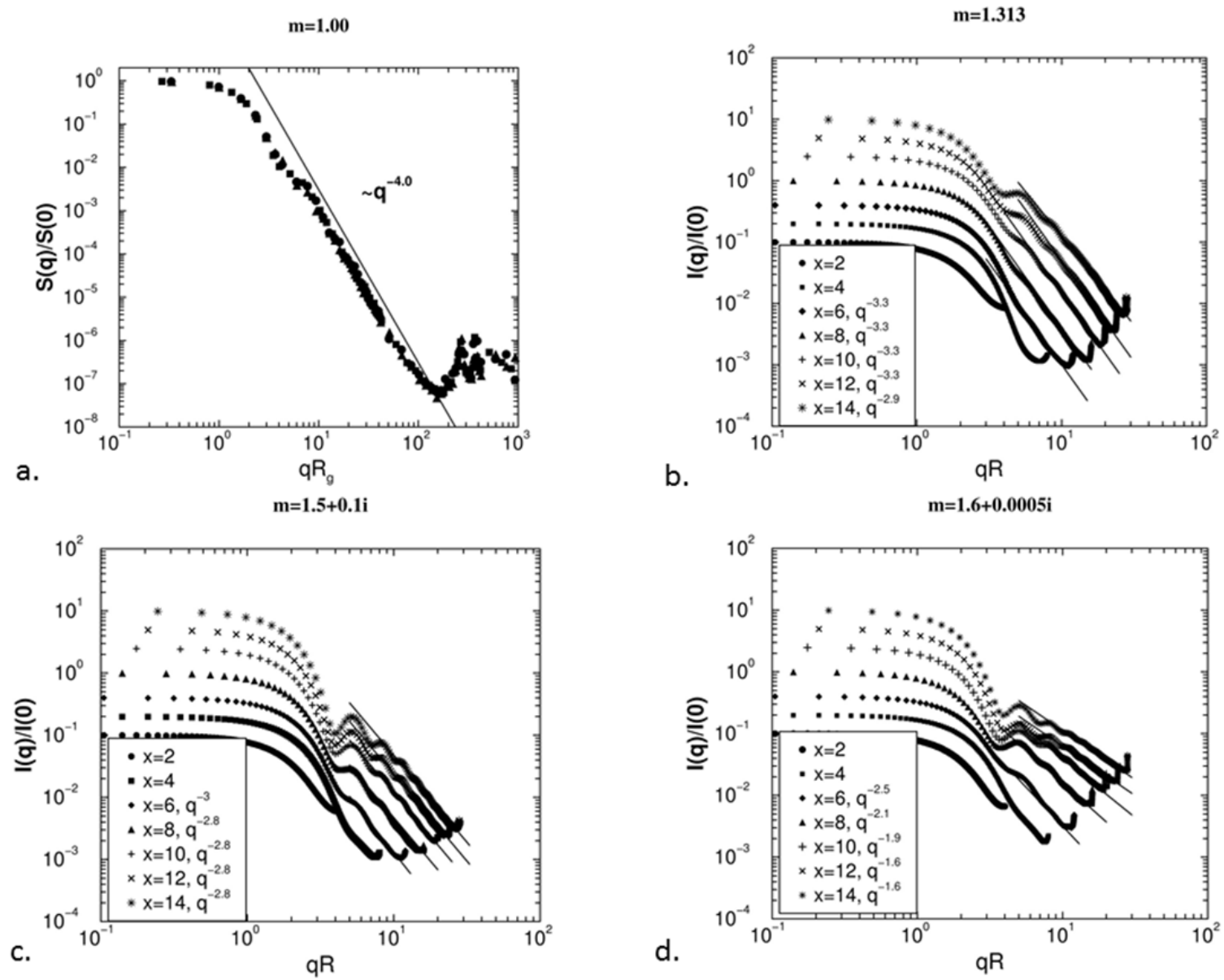

Rayleigh-Debye-Gans limit, the structure factor shows the Porod exponent of -4 .

Figure 3. Q-space analysis of the scattering by pocked spheres. Part a shows the $\mathrm{m} \rightarrow 1.00$, Rayleigh-Debye-Gans limit, the structure factor. Parts $\mathrm{b}$, c and $\mathrm{d}$ are with refractive indices, $\mathrm{m}$, as marked for a range of size parameters, $\mathrm{x}$. Thin straight lines represent power laws with $\mathrm{q}$ as described in the legend. Plots are intensity shifted to avoid overlap. 
3.4 Agglomerate debris. The Q-space plots for agglomerate debris are shown in Fig. 4. Similar to the strongly damaged and pocked sphere cases above, Fig. 4 again shows all six features described above including Rayleigh, Guinier and definite power law regimes. Figure 4a shows the square of the Fourier transform of the real space structure for three of the agglomerated debris particles. Once again in the Rayleigh-Debye-Gans limit, the Porod exponent of -4 is found.
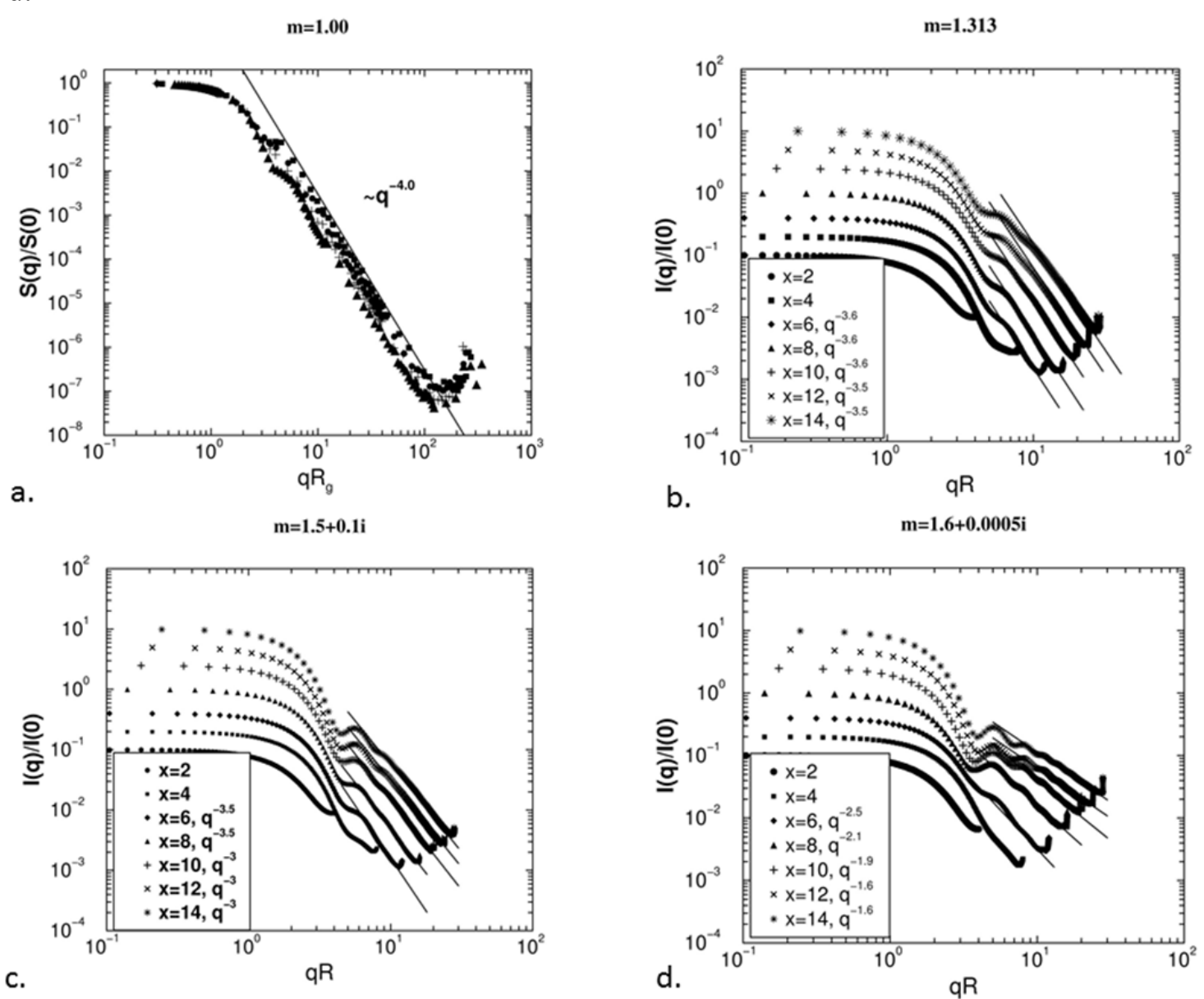

d.

qR

Figure 4. Q-space analysis of the scattering by agglomerated debris particles. Part a shows the $\mathrm{m} \rightarrow 1.00$, Rayleigh-Debye-Gans limit, the structure factor. Parts $\mathrm{b}, \mathrm{c}$ and $\mathrm{d}$ are with refractive indices, $\mathrm{m}$, as marked for a range of size parameters, $\mathrm{x}$. Thin straight lines represent power laws with $\mathrm{q}$ as described in the legend. Plots are intensity shifted to avoid overlap.

3.5 Summary of power law exponents. The figures above show the same Porod exponent of -4 for all the $\mathrm{m} \rightarrow 1.00$, Rayleigh-Debye-Gans limit, the structure factors. Most generally, this limit applies when the phase shift parameter $\rho<1$, and by Eq. 2, $\rho$ is a function of both the size parameter and the refractive index. The figures above also show different exponents with non- 
unity refractive indices for particles with larger size parameters. This leads to the question: how do the exponents evolve away from the RDG limit with increasing phase shift parameter $\rho$ ? To answer this question the exponents are plotted in Fig. 5 as a function of $\rho$. We remark that the power law exponents are based on our visual estimates of lines that best fit our estimate of the power law regimes in the plots. Thus they should be considered as semi-quantitative. With this caveat, Fig. 5 shows a general trend of exponents decreasing with increasing $\rho$. Similar plots versus refractive index or size parameter show a similar trend, but the data are more spread out. This implies that $\rho$ yields the most universal description of the decreasing exponents.

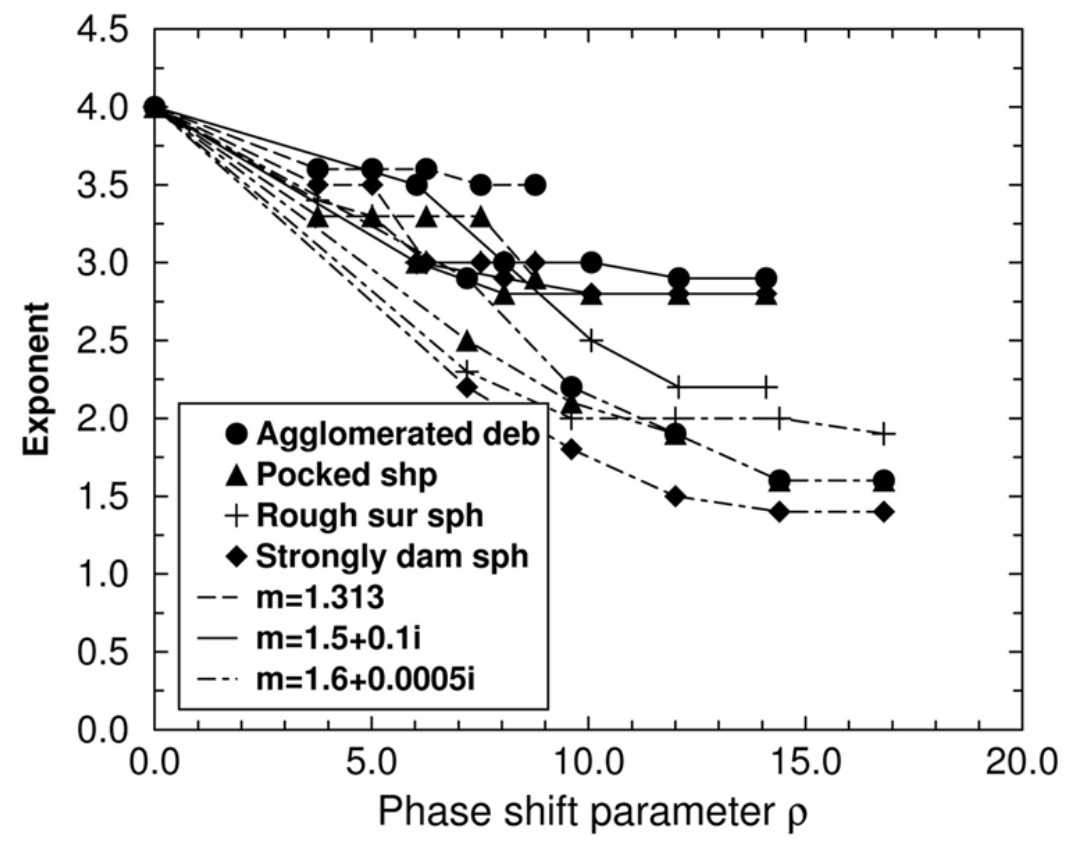

Figure 5. Power law exponents for the various sphere types as a function of phase shift parameter $\rho$.

3.6 Forward scattering. To set the context for a study of the forward scattering properties of these irregularly shaped particles, recall the Rayleigh scattering cross section for polarized light [11]

$$
\mathrm{C}_{\text {sca }}=(8 \pi / 3) \mathrm{k}^{4} \mathrm{R}^{6} \mathrm{~F}(\mathrm{~m})
$$

where

$$
\mathrm{F}(\mathrm{m})=\left|\left(\mathrm{m}^{2}-1\right) /\left(\mathrm{m}^{2}+2\right)\right|^{2}
$$



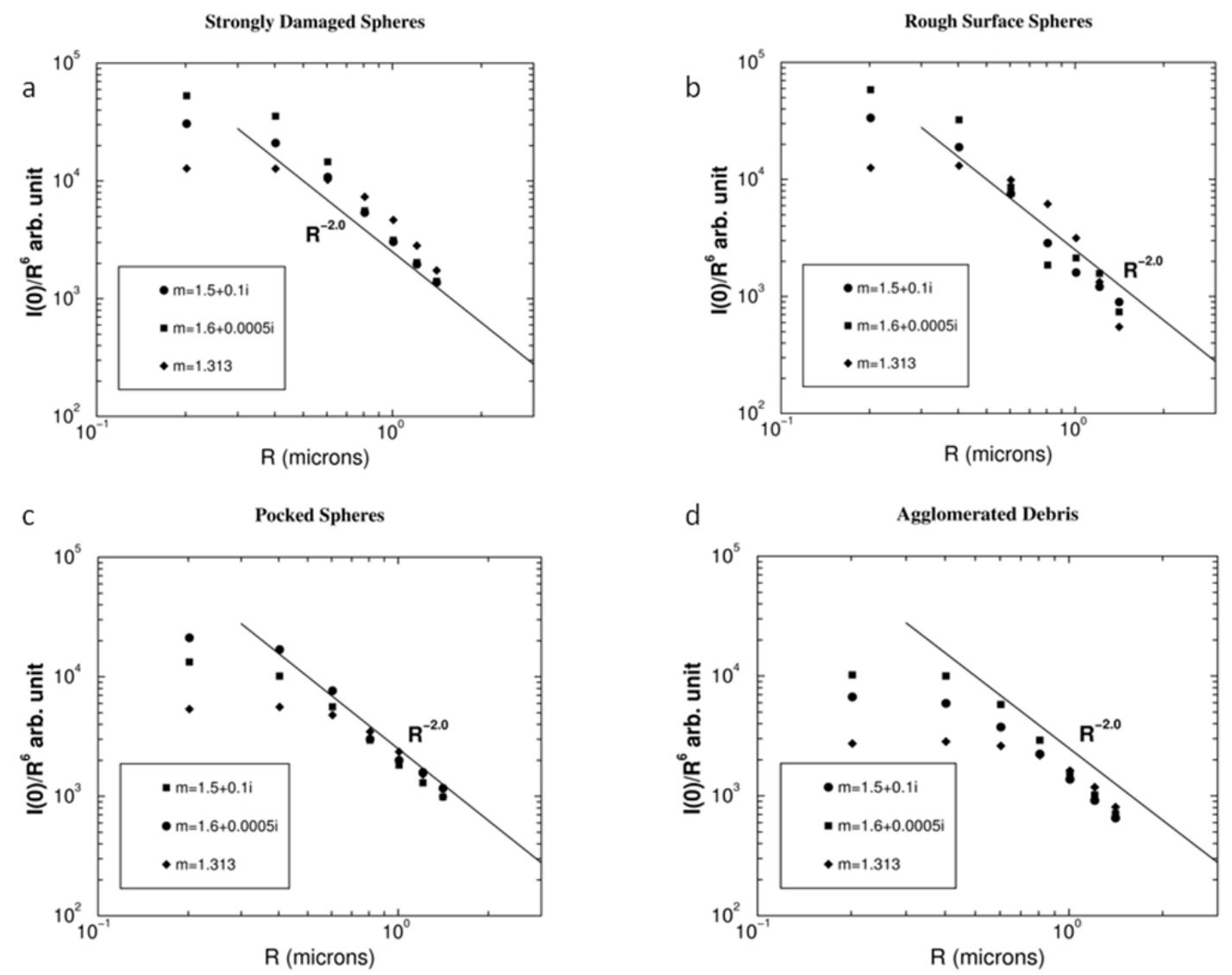

Figure 6. Forward scattering intensity divided by the sixth power of the particle radius as a function of particle radius for all four particle types and all three refractive indices.

Figure 6 plots the forward scattering intensity normalized by the sixth power of the particle radius, $I(0) / R^{6}$, as a function of $R$ for all the particles. For $R \leq 0.4 \mu \mathrm{m}$ the figure shows that the forward scattering has a sixth power functionality with the radius, $R^{6}$, for $m=1.313$ and the trend for the other two refractive indices is consistent with this conclusion. Furthermore for small sizes there is a strong refractive index dependence. For $\mathrm{R}>0.4 \mu \mathrm{m}$ the size dependence crosses over to $\mathrm{R}^{4}$ and the refractive index dependence weakens. The value of the phase shift parameter at this crossover near $\mathrm{R}=0.4 \mu \mathrm{m}$ is in the range of $\rho \approx 2.4$ to 4.8 , depending on the refractive index.

Figure 7 shows $\mathrm{I}(0)$ versus the Lorentz term, Eq. (4), for $\mathrm{R}=0.2 \mu \mathrm{m}$, the smallest particle size of all four particle types. The functionality is linear with some deviations. 
These size and refractive index functionalities are the same as those for spherical particle Rayleigh scattering, Eqs. (3) and (4). This and the lack of q, hence $\theta$, dependence in the forward lobe, imply that the forward scattering lobe of these irregularly shaped particles obeys Rayleigh scattering for sizes $\mathrm{R}<0.4 \mu \mathrm{m}$. All this behavior occurs for Mie scattering by spheres as well [2].

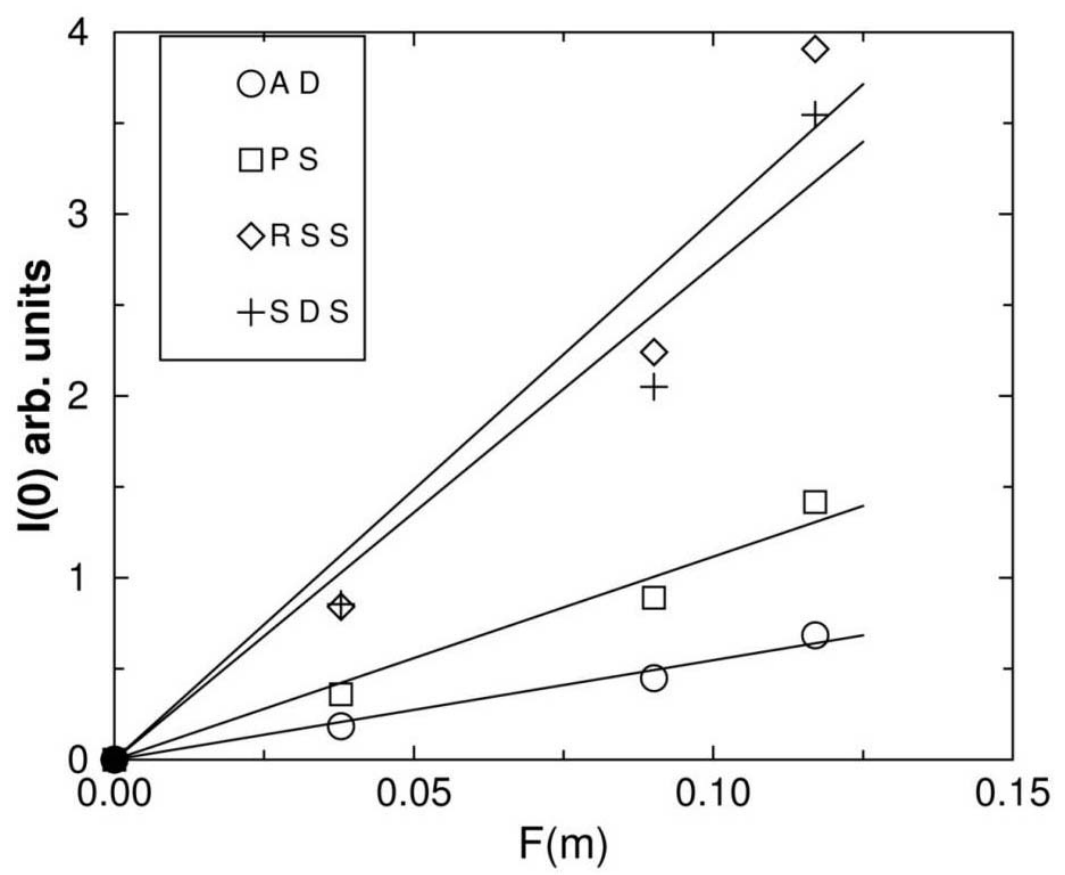

Figure 7. Forward scattered intensity versus $F(m)=\left|\left(m^{2}-1\right) /\left(m^{2}+2\right)\right|^{2}$ for all for particle types (AD, agglomerated debris; PS, pocked spheres; RSS, rough surface spheres; SDS, strongly damaged spheres) and a radius of $\mathrm{R}=$ $0.2 \mu \mathrm{m}$. Lines are best fits to the data points including the origin.

\section{Discussion}

The Q-space analysis has uncovered power law functionalities when $\mathrm{qR}>3.5$ with quantifiable exponents for the scattering phase function for four different irregularly shaped particles. It also emphasizes the fact that scattering from all particles has an angle independent forward scattering lobe when $\mathrm{qR}<1$ and a Guinier crossover near $\mathrm{qR} \approx 1$. These properties are characteristics of spheres, spheroids, fractal aggregates and dusts as well.

All the particles studied here were perturbations of solid spheres. The phase shift parameters for all the particles were in the range $1.2 \leq \rho \leq 16.8$, hence $\rho>1$. Previous studies of Mie scattering by spheres $[1,2,4]$ show that the exponents of the power laws evolve from the 
Porod value of -4 at the $\rho \rightarrow 0$ limit, to two power laws with increasing $q R$ of -2 and -4 separated by the $\rho$-crossover at ca. $\mathrm{qR} \approx 1.2 \rho$. For the irregular spheres studied here the $\rho \rightarrow 0$ limit is also described by a single power law with the same Porod exponent -4 . However, once $\rho>1$, two power laws were never seen, although the cross over is only distinctive in normal spheres when $\rho$ $>10$, a condition that only marginally applies to some of the irregular spheres here. Instead the power law exponents decreased with increasing $\rho$. Thus the randomization of the sphere's structure to create these irregular particles seems to have eliminated the $\rho$-crossover and led to exponents usually different than those found for spheres when $\rho>1$.

A general treatment of the Porod exponent shows that it is equal to the negative of $2 \mathrm{D}_{m}-$ $D_{s}$ where $D_{m}$ is the mass fractal dimension and $D_{s}$ is the surface fractal dimension of the particle [17]. For a sphere $D_{m}=3, D_{s}=2$ so $2 D_{m}-D_{s}=4$. For the irregularly shaped particles in this study, the RDG limit $(\rho \rightarrow 0)$ found a Porod exponent of -4 . Thus we conclude that the mass and surface fractal dimensions of these perturbed spheres remain equal to 3 and 2, respectively, despite the structural randomization process.

The forward scattering lobe obeys, or is trending to obey, Rayleigh scattering in both the refractive index and particle volume dependence. This result has been found to be true for spheres [2] and fractal aggregates [8] as well. When $\rho \geq 4$, the functionality on the refractive index is severely diminished, and the size dependence falls from $R^{6}$ to $R^{4}$. This same behavior occurs for spheres.

\section{Conclusion}

This paper applied the Q-space analysis method to the calculated scattering phase function of four irregularly shaped particles. This analysis showed that the scattering can be described by a series of universal features as a function of the dimensionless qR: At small $q$ the scattered intensity in the forward lobe obeys the Rayleigh scattering functionalities on refractive index and particle volume and is constant (angle independent) as $\mathrm{q}$ approaches 0 . This is followed by a Guinier regime near $\mathrm{qR} \approx 1$. For $\mathrm{qR}>3.5$ the scattered intensity falls off with a power law with quantifiable negative exponents with absolute values less than 4; although the power law description is marginal for the rough surface spheres. When $\mathrm{m} \rightarrow 1.00$ the Porod exponent of -4 occurs for all the particles. The power law exponents decrease with increasing phase shift parameter, $\rho$. Many of these universal features have been observed for aggregates, spheres, spheroids and dusts. Thus Q-space analysis can yield a simple, comprehensive and quantitative description of the scattered intensity for a wide variety of shapes and should be used in the analysis of scattering phase functions.

Acknowledgment. We thank Gorden Videen for suggesting our collaboration. We also thank an anonymous referee whose steadfast criticisms led us to improve our manuscript.

\section{References}

[1] Sorensen CM, Fishbach JD. Patterns in Mie scattering. Opt Commun 2000;173:145-53.

[2] Berg MJ, Sorensen CM, Chakrabarti A. Patterns in Mie scattering: evolution when normalized by the Rayleigh cross section. Appl Opt 2005; 44(34): 7487-93.

[3] Berg MJ. Power-law patterns in electromagnetic scattering: A selected review and recent 
progress. J Quant Spectrosc Radiat Transfer (2012) http://dx.doi.org/10.1016/j.jqsrt.2012.05.015. [4] Sorensen CM. Q-Space Analysis of Scattering by Particles. J Quant Spectrosc Radiat Transfer, submitted.

[5] Zubko E, Shkuratov Y, Kiselev NN, Videen G. DDA simulations of light scattering by small irregular particles with various structure, J Quant Spectrosc Radiat Transfer. 2006; 101: 416434.

[6] Zubko E, Shkuratov Y, Mishchenko MI, Videen G. Light scattering in a finite multi-particle system, J Quant Spectrosc Radiat Transfer 2008;109: 2195- 2206.

[7] Zubko E, Kimura H, Shkuratov Y, Muinonen K, Yamamoto T, Okamoto H, Videen G. Effect of absorption on light scattering by agglomerated debris particles J Quant Spectrosc Radiat Transfer. 2009;110: 1741- 1749 .

[8] Sorensen CM. Light scattering by fractal aggregates: a review. Aerosol Sci Technol 2001; 35: $648-87$.

[9] Guinier A, Fournet G, Walker CB, Yudowitch KL. Small-angle scattering of X-rays. New York: John Wiley; 1955.

[10] Mie G. Beitrage zur optik truber medien speziel kolloidaler metallosungen. Ann. Phys. 1908; 25: 377-445.

[11] van de Hulst HC. Light scattering by small particles. New York: Dover; 1981.

[12] Sorensen CM. Q-space analysis of scattering by dusts. J Quant Spectrosc Radiat Transfer. 2013; 115: 93- 95 (2013).

[13] Volten H, Munoz O, Rol E, de Haan JF, Vassen W, Hovenier JW, Muinonen K, Nousiainen $\mathrm{T}$, Scattering matrices of mineral aerosol particles at $441.6 \mathrm{~nm}$ and $632.8 \mathrm{~nm}$. J Geophys Res 2001; 106: 17375-17401.

[14] Munoz O, Volten H, Hovenier JW, Nousiainen T, Muinonen K, Guirado D, Moreno F, Waters LBFM, Scattering matrix of large Saharan dust particles: Experiments and computations.

J Geophys Res 2007; 112:D13215.

[15] Draine BT, Flatau PJ. The discrete dipole approximation for scattering calculations. J Opt Soc Am A 1994;11:1491-9.

[16] Porod G. Die Rontgonkleinwinkelstreuung von Dichtgepacken Kolloiden

Systemen, Kolloid Z. 1951; 124:83-114.

[17] Oh C, Sorensen CM, Scaling Approach for the Structure Factor of a Generalized System of Scatterers. J. Nanopart. Res. 1999; 1: 369-377. 